\title{
Sensitization to Common Allergens Among Children with Asthma and Allergic Rhinitis in Qatar
}

This article was published in the following Dove Press journal: Journal of Asthma and Allergy

\author{
Khalid Zahraldin (D) \\ Prem Chandra ${ }^{2}$ \\ Amjad Tuffaha $\mathbb{I D}^{3,4}$ \\ Mohammad Ehlayel (D) ${ }^{4,5}$ \\ 'Section of Pediatric Pulmonology, \\ Department of Pediatrics, Hamad Medical \\ Corporation, Doha, Qatar; ${ }^{2}$ Department \\ of Statistics, Medical Research Center, \\ Hamad Medical Corporation, Doha, \\ Qatar; ${ }^{3}$ Division of Pediatric \\ Pulmonology, Sidra Medicine, Doha, \\ Qatar; ${ }^{4}$ Department of Pediatrics, Weill- \\ Cornell-Medical College- Qatar, Ar- \\ Rayan, Qatar; ${ }^{5}$ Section of Pediatric \\ Allergy-Immunology, Department of \\ Pediatrics, Hamad Medical Corporation, \\ Doha, Qatar
}

Background: Childhood asthma (A) and allergic rhinitis (AR) are common in Qatar. Aeroallergens sensitization is integral in disease pathogenesis and clinical presentation. Determining sensitization patterns assists clinicians in tailoring an efficient medical management.

Objective: To determine the aeroallergen sensitization pattern and relationship to clinical parameters.

Methods: A retrospective review of children (2-14-years) files with i) Pediatric Allergist/or Pulmonologist confirmed-diagnosis of A, and AR, and ii) positive skin prick test (SPT).

Results: Among 473 patients (69.1\% males; 30.9\% females), aged 7.6 years, family history was positive in $66.3 \%$ : $59.4 \%$ in A, $64.2 \% \mathrm{AR}$, and $78.2 \% \mathrm{~A}-\mathrm{AR}$. The number of allergens/ patients was $2.1 \pm 1.7$. Median eosinophil count was 400 cells/ul and $\operatorname{IgE} 287 \mathrm{KU} / \mathrm{L}$. Rates of $\mathrm{A}, \mathrm{AR}$, and A-AR varied significantly in children $\leq 5$ years compared to $>10$ years: A was $43.2 \%$ vs $17.8 \%$, and AR $34.5 \%$ vs $16.4 \%$. Two hundred and four children $(43.1 \%)$ were mono-sensitized, 215 (45.5\%) oligosensitized (2-3 allergens), and 54 (11.4\%) polysensitized ( $\geq 4$ allergens). A-AR ranked the top number of positive allergens. The commonest aeroallergen was Der p1 (38.1\%), followed by Der f (29.0\%), cat (22.6\%), alternaria (18.8\%), American cockroach (18.4\%), and dog (14.0\%). House dust mite (HDM) and American cockroach were commoner in $\leq 5$ years than older $>10$-year children $(52.5 \%, 24.1 \%)$, while cat and dog allergens were commoner in older ones $(37.1 \%, 21.6 \%)$.

Conclusion: Family history is quite positive in patients with A and AR. Common aeroallergens include HDM, cats, and alternaria in the young children, while animal allergens were commoner in the older children.

Keywords: asthma, allergic rhinitis, skin prick test, children

\section{Introduction}

The prevalence of respiratory allergic disorders has increased in the last few decades and has become a major medical concern, and the impact of these diseases is huge on patients, families, and societies. ${ }^{1,2}$

Despite decades of research, little is known about which factors are the main determinants of the high and of the large global variation in asthma and other respiratory allergic disease prevalence. ${ }^{3}$ By the time children reach middle school (10-13 years of age), asthma is very strongly associated with common inhalant allergens. ${ }^{4}$ At this age, the odds ratio for wheezing among sensitized compared to non-sensitized individuals is often 6 folds or higher ${ }^{5,6}$.
Correspondence: Mohammad Ehlayel Section of Pediatric Allergy-Immunology, Department of Pediatrics, Hamad Medical Corporation, PO Box 3050, Doha, Qatar Tel +97444392834

Fax +97444439571

Email mehlayel@gmail.com 
SPT with the standardized allergen panels is highly recommended for epidemiological studies, clinical use, and research. ${ }^{7}$ It is essential to determine the patterns of aeroallergen sensitization for purposes of disease management in a particular region (eg, Gulf area) where aeroallergen monitoring systems have not been well established. Although respiratory allergies are common in Qatar, both in children and adults, ${ }^{8-12}$ the medical research is sparse on the pattern of aeroallergens, particularly in allergic children. ${ }^{10}$

The aim of this study is to determine the prevalence of aeroallergen sensitization, rate of specific aeroallergen sensitization, and pattern change in this sensitization over a 6-year period in children with respiratory allergic disorders in Qatar.

\section{Methods}

\section{Study Design and Subjects}

In this retrospective chart review of children who were evaluated at the pediatric allergy and pediatric pulmonology clinics, Hamad General Hospital, a tertiary care hospital and affiliated with Weill-Cornell Medical College-Qatar in Doha, Qatar between September 2008 and October 2014. Using the clinic logbook, we collected data on i) children 2-14-years old with ii) physician-confirmed-diagnosis of $\mathrm{A}$, and $\mathrm{AR}$, and ii) and positive SPT to at least one aeroallergen.

The study population comprised all patients, between 2 and 14 years, that underwent skin prick testing for aeroallergens during the study period. We excluded patients who had i) severe debilitating chronic illnesses (eg, chronic renal failure, hepatic failure, malignancy, etc.), ii) dermatographism as per physical examination prior to SPT, iii) patients on immunotherapy (subcutaneous or sublingual immunotherapy), or patients on iv) chronic systemic steroids at the time of SPT or immune-modulator therapy (eg, omalizumab or similar). The study was conducted in accordance with the Declaration of Helsinki, and the protocol was approved by the Institutional Review Board (IRB) at Hamad Medical Corporation. As this was a retrospective study, informed consent from patients is not required by the Institutional Review Board(IRB) of Hamad Medical Corporation. The privacy of all participants was protected, we confirmed the data were anonymized and maintained with confidentiality.

\section{SPT Technique and Extract}

We utilized a standardized panel of SPT at our clinics for all patients suspected of sensitization to aeroallergens and it included 12 common aeroallergens: A. alternata, A. fumigatus, C. herbarum, feather mix, grass mix, penicillium, rhizopus, cat dander, dog hair, D pteronyssinus (Der p1), D farinae (Der f), and cockroaches. SPTs to other aeroallergens were performed, when indicated by medical history, including alfalfa, palm date, mesquite, B. tropicalis, acacia, birch, and mugwort. SPTs were performed by a well-trained allergy/pulmonary function laboratory technician under the supervision of the clinic allergists/pulmonologists using a standardized technique. A drop of each aeroallergen extracts along with a drop from the positive (histamine) and negative (normal saline) controls was applied to the anterior forearm skin and then skin pick through the drop using Stallerpoint ${ }^{\circledR}$ plastic lancets. The test was read by the technician and the clinic allergists/ pulmonologists 10 to 15 minutes after the application of the allergen. A positive test was defined as a wheal of at least $3 \mathrm{~mm}$ larger than the negative control. Mono-sensitization was defined as sensitization to one aeroallergen of the SPT panel, oligo-sensitization as sensitization to $2-3$, and polysensitization as sensitization to $\geq 4$ aeroallergens.

\section{Data Collection}

Basic demographic data (age, sex), type of respiratory allergic disease, family history of allergies, and SPT results were collected and entered into an MS Excel spreadsheet and analyzed in all patients. Whenever available, CBC with differential counts and total serum IgE were collected; too.

\section{Statistical Analysis}

All collected data were analyzed using statistical packages SPSS 24.0 (SPSS Inc. Chicago, IL) and Epi-info (Center for Disease Control and Prevention, Atlanta, GA) software. The normally distributed data and results were presented with mean and standard deviation (SD) with corresponding 95\% confidence interval (CI); the remaining results were reported with median and inter-quartile range (IQR). Categorical data were summarized using frequencies and percentages. Associations between two or more qualitative variables were assessed using Pearson Chi-square test $\left(\mathrm{X}^{2}\right)$ or Fisher exact Chi-square or Yates corrected Chi-square tests as appropriate. All $\mathrm{P}$ values presented were two-tailed, and $P$ values $<0.05$ were considered as statistically significant.

\section{Results}

A total of 473 children ( $\leq 14$ years) with at least one respiratory tract allergic disease and SPT showing sensitivity to at least one allergen were enrolled in the study. Mean age $( \pm \mathrm{SD})$ of the study cohort was $7.6 \pm 3.3$ years and $69.1 \%$ were males (Table 1 ). 
Table I Clinical Characteristics of 473 Children with Respiratory Allergic Diseases

\begin{tabular}{|l|l|}
\hline Variable & \\
\hline Total number ( $\mathrm{n}=473$ patients) & \\
\hline Age (years \pm SD) & $7.6 \pm 3.3$ \\
\hline Sex & \\
Male & $327(69.1 \%)$ \\
Female & $146(30.9 \%)$ \\
M/F ratio & $2.2 / 1.0$ \\
\hline Allergy Type ( $=473$ patients) & \\
Asthma & $175(37 \%)$ \\
Allergic rhinitis & $57(12 \%)$ \\
Both & $209(44.2 \%)$ \\
Others** & $32(0.8 \%)$ \\
\hline Family history of allergies*, ( $n=406$ patients) & \\
Positive & $269(66.3 \%)$ \\
Negative & $137(33.7 \%)$ \\
\hline No of positive SPT (mean $\pm S D)$ & $2.1 \pm 1.7$ \\
\hline
\end{tabular}

Notes: *Data available on 406 patients. **Others: A or AR coexistent with other non-respiratory allergic diseases: drug allergy, eosinophilic esophagitis, etc.

The rates of $\mathrm{A}, \mathrm{AR}$, or A-AR, in addition to family history of these diagnoses, were significantly different between different age groups, Table 2 .

The number of allergen sensitization per patient was $2.1 \pm 1.7$ (95\% CI: 1.93-2.24) allergens. Two hundred and four children (43.1\%) were mono-sensitized, 215 (45.5\%) oligosensitized and 54 (11.4\%) polysensitized. Almost $90 \%$ were positive for $\leq 3$ allergens, $75 \%$ for $\leq 2$ allergens, and around $40 \%$ for one allergen as in Figure 1.

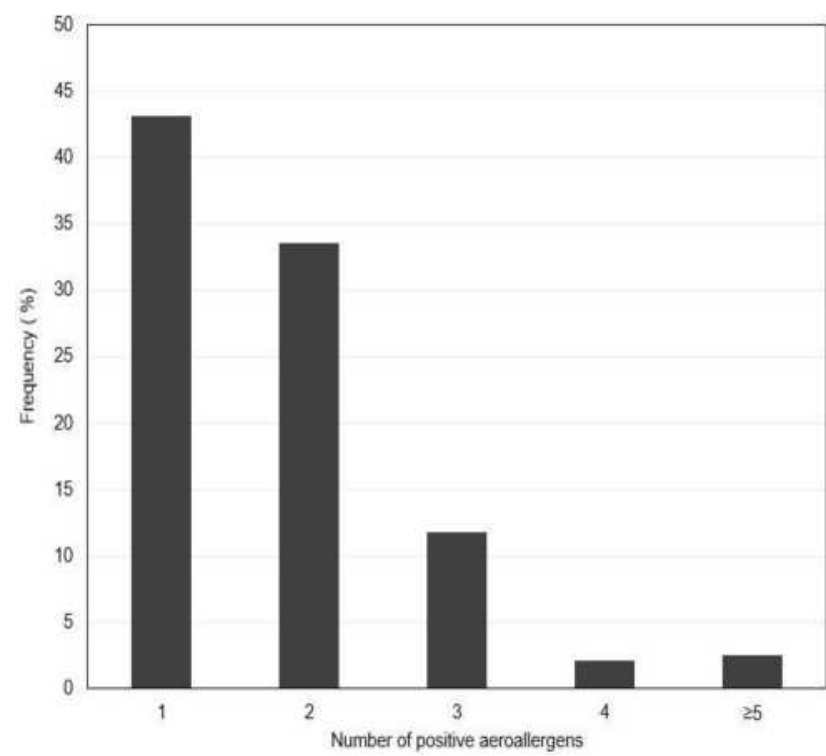

Figure I Frequency of positive skin tests among children with respiratory allergy.

The number of polysensitized children $(\mathrm{n}=271,57.3 \%)$ was more than monosensitized $(n=202,42.7 \%)$ ones. However, rates of monosensitization were comparable to those of polysensitization for children $\leq 5$ years ( 30.7 vs $30.3 \%$ ), children $6-10$ years' age ( $48 \%$ vs $49.8 \%$ ), and those $10>$ years $(21.3 \%$ vs $19.9 \%)$.

Compared to A alone, AR alone, or other disease combinations, A-AR ranked the highest rates of positive SPT regardless of number of positive allergens, data not shown.

The frequency of specific allergens across all age groups: Der p1 observed in 180 subjects (38.1\%), Der f 137 (29.0\%), cat allergen 107 (22.6\%), A. alternata 89 (18.8\%), American

Table 2 Rates of Different Types of Respiratory Allergic Disease According to Sex, Age, and Family History of Allergic Diseases

\begin{tabular}{|c|c|c|c|c|c|}
\hline \multirow[t]{2}{*}{ Characteristics } & \multicolumn{4}{|c|}{ Number of Subjects ( $n=473$ Patients) } & \multirow[t]{2}{*}{$P$ value } \\
\hline & Asthma & (A) Rhinitis & Both (A-AR) & Others** & \\
\hline \multicolumn{6}{|l|}{ Sex } \\
\hline Male & II 14 (28.7\%) & $35(10.7 \%)$ & $158(38.3 \%)$ & $20(6.3 \%)$ & 0.032 \\
\hline Female & $61(4 \mid .8 \%)$ & $24(16.4 \%)$ & $50(34.2 \%)$ & $\mathrm{II}(7.5 \%)$ & \\
\hline \multicolumn{6}{|l|}{ Age } \\
\hline $2-5 y r$ & $76(43.4 \%)$ & $20(35.1 \%)$ & $39(18.8 \%)$ & $9(29.0 \%)$ & \\
\hline$>5 y r-10 y r$ & $68(38.9 \%)$ & $28(49.1 \%)$ & $|2|(57.9 \%)$ & $17(51.6 \%)$ & $<0.0001$ \\
\hline$>10 y r-14 y r$ & $31(17.7 \%)$ & $9(16.4 \%)$ & $49(23.3 \%)$ & $6(19.4 \%)$ & \\
\hline \multicolumn{6}{|c|}{ Family history of allergy* } \\
\hline Positive & $85(59.4 \%)$ & $34(64.2 \%)$ & $147(78.2 \%)$ & $3(13.6 \%)$ & $<0.0001$ \\
\hline Negative & $58(40.6 \%)$ & $19(35.8 \%)$ & $4 \mathrm{I}(2 \mathrm{I} .8 \%)$ & $19(86.4 \%)$ & \\
\hline
\end{tabular}

Notes: Yates corrected Chi-square test was applied in case of small cell frequencies $(50 \%$ or more cells have expected frequencies $<5)$ to compute respective statistical P-value. *Data available on 406 patients. **Others: A or AR coexistent with other non-respiratory allergic diseases: drug allergy, eosinophilic esophagitis, etc. 


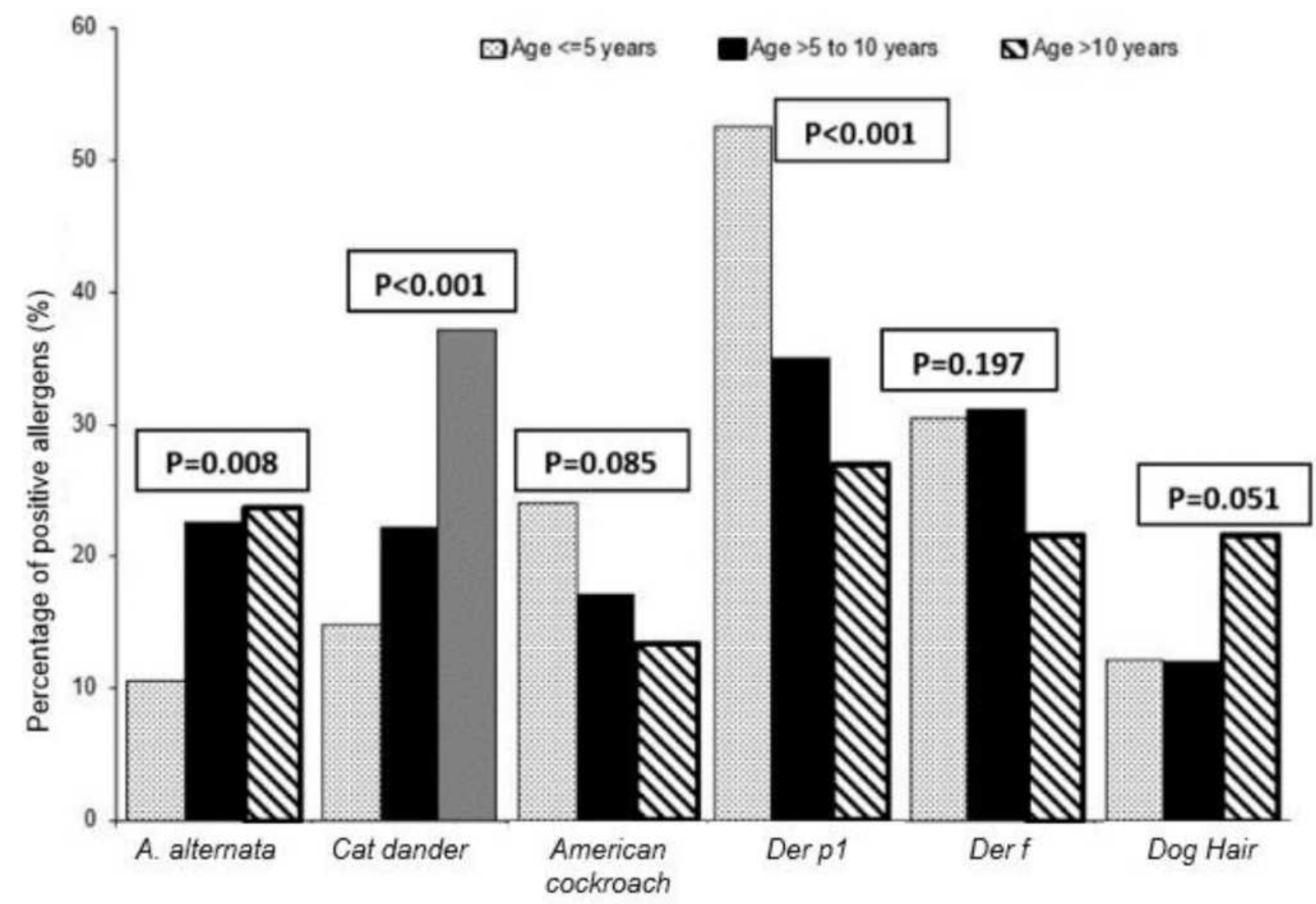

Type of Allergen

Figure 2 Rates of different types of allergens according to age groups of children with respiratory allergic diseases.

cockroach 87 (18.4\%), dog allergen 66 (14.0\%), C. herbarum 40 (8.5\%), grass mix 40 (8.5\%), A. fumigatus 25 (5.3\%), and feather mix $13(2.7 \%)$. HDM tended to be the most common allergen for all age groups. It was noticed that HDM and American cockroach sensitization rates were highest among children less than 5 years and tended to be less frequent in the older age groups. Rates of cat and dog allergens were higher in children $>10$ years. However, rates of alternaria were higher to older children (6-14 years) than those in young ( $<5$ years) children, as in Figure 2. Median (25th-75th percentiles) absolute eosinophil count of 400 cells/ul (180-738), and serum IgE $287 \mathrm{KU} / \mathrm{L}$ (112-852).

\section{Discussion}

Sensitization to aeroallergens is commonly associated with AR and asthma, yet numerous studies have shown that the distribution and pattern of allergy differ significantly from one country to another. Identifying common allergens in local populations is imperative for diagnosis and targeted specific therapy. ${ }^{13}$ Our study was conducted with the aim of specifically targeting the pediatric population and as far as we know, it might be the first study to solely include children under the age of 14 in Qatar. This discussion will include further elaboration in regards to the most common aeroallergens in patients with $\mathrm{A}, \mathrm{AR}$, or $\mathrm{A}-\mathrm{AR}$ and comparison with other studies in the region.

Bronchial asthma and AR tended to be more common in females than males with the percentages of $41.8 \%$ and $16.4 \%$, respectively. However, the results were not statistically significant. One similar study on 1106 asthmatic adult patients in Doha, State of Qatar was established in 2003 and showed similar results, where asthma was more prevalent in females $32.7 \%$ than males $29.8 \%{ }^{8}$

In this study, the rate of AR alone, asthma alone, or AAR and asthma were significantly more common in the 
young age groups (less than 10 years) compared to older counterparts (those above 10 years).

Asthma is a multifactorial disease that can be influenced by environmental and genetic factors. Family history plays an important role in the pathogenesis of the disease. Therefore, identifying people with a family history may promote their earlier diagnosis and treatment. ${ }^{14}$ In our study, there was significant difference in rates of positive family history compare to negative family history children with A (1.5 folds), AR (1.8 folds), but highest rates among those with A-AR (3.6x folds). The rates of positive family history were significantly different in the 3 allergic respiratory diseases: for A 59.4\%, AR 64.2\%, and A-AR $78.2 \%$. Similarly, these findings were comparatively approximate to the previous study conducted in Qatar which showed that $44 \%$ of asthmatic patient had a strong positive family history. ${ }^{8}$

In this study, the most common aeroallergen was Der $\mathrm{p} 1$ (38.1\%), followed by Der f (29.0\%), cat (22.6\%), A. alternata (18.8\%), American cockroach (18.4\%), dog (14.0\%), C. herbarum (8.5\%), grasses (8.5\%), A. fumigatus (5.3\%), and feathers $(2.7 \%)$. Similar results were observed in previous studies done in Qatar and Saudi Arabia. ${ }^{8,15-17}$ On the other hand, a study in UAE that involved 180 pediatric patients with allergies, showed that the most common aeroallergens were Der f (38.3\%) followed by Der p1 (37.2\%) and Cat dander (17.2\%). ${ }^{13}$ Moreover, there was a study in Jordan and two previous studies directed in Iran (one of them was a meta-analysis study) revealed that the most common aeroallergens were pollens followed by HDM and cockroach. ${ }^{18-20}$ The differences between the results from Gulf states, including ours, with those from Jordan and Iran might be related to genetic, environmental, or geographical/ meteorological factor or even lifestyles.

HDM and American cockroach sensitization rates were commoner in children $<5$ years $(52.5 \%, 24.1 \%)$ than older children, while rates for cat and dog allergen sensitization $(37.1 \%, 21.6 \%)$ were highest in children older than 10 years. These results can be explained by the assumption that younger children stay more indoor and therefore are more exposed to HDM and cockroaches than older children who play outside baring themselves to animal allergens. Similar conceptual results were found in the following studies: Riyadh (HDM and aspergillus sensitization rates were more common in adults whereas Salsola kali and date palm sanitization were more common in children), ${ }^{16}$ Iran (cockroaches sanitization is more prevalent in children $<16$ years, whereas
HDM, pollens, cat dander, and molds were more common in adults $>16$ years), ${ }^{20}$ Jordan (cats and HDM are more common in younger age group $)^{18}$ and Saudi Arabia (where HDM is the most prevalent allergen in younger age group). ${ }^{17}$

\section{Conclusions}

It has come to our conclusion that asthma and allergic rhinitis are strongly correlated with a positive family history. Moreover, in comparison to the older age group, the younger age groups had a higher rate of allergic diseases with HDM, cats, and alternaria being the most common amongst this group and animal allergens the most common amongst the older age group. The majority of children tested positive to 4 inhalant allergens.

This study presents evidence that family history still remains of great help when clinically diagnosing asthma and allergic rhinitis, and in addition to other factors, child's age should be considered when selecting the aeroallergens panel for skin testing.

\section{Acknowledgments}

We acknowledge Hamad Medical research Centre for approving and funding our study. Research Proposal $14,352 / 14$.

\section{Disclosure}

The authors declare no conflicts of interest for this work.

\section{References}

1. Reed SD, Lee TA, McCrory DC. The economic burden of allergic rhinitis: a critical evaluation of the literature. Pharmacoeconomics. 2004;22(6):345-361. doi:10.2165/00019053-200422060-00002

2. Schoenwetter WF, Dupclay L, Appajosyula S, Botteman MF, Pashos CL. Economic impact and quality-of-life burden of allergic rhinitis. Curr Med Res Opin. 2004;20(3):305-317. doi:10.1185/ 030079903125003053

3. Asher MI, Montefort S, Björkstén B, et al. Worldwide time trends in the prevalence of symptoms of asthma, allergic rhinoconjunctivitis, and eczema in childhood: ISAAC phases one and three repeat multicountry cross-sectional surveys. Lancet. 2006;368(9537):733-743. doi:10.1016/S0140-6736(06)69283-0

4. Sporik R, Holgate ST, Platts-Mills TA, Cogswell JJ. Exposure to house-dust mite allergen (Der p I) and the development of asthma in childhood. A prospective study. N Engl J Med. 1990;323(8):502-507. doi:10.1056/NEJM199008233230802

5. Peat JK, Tovey E, Toelle BG, et al. House dust mite allergens. A major risk factor for childhood asthma in Australia. Am J Respir Crit Care Med. 1996;153(1):141-146. doi:10.1164/ajrccm.153.1.8542107

6. Halonen M, Stern DA, Wright AL, Taussig LM, Martinez FD. Alternaria as a major allergen for asthma in children raised in a desert environment. Am J Respir Crit Care Med. 1997;155 (4):1356-1361. doi:10.1164/ajrccm.155.4.9105079 
7. Heinzerling L, Burbach G, Edenharter G, et al. GA 2 LEN skin test study I: GA ${ }^{2} L E N$ harmonization of skin prick testing: novel sensitization patterns for inhalant allergens in Europe. Allergy. 2009;64 (10):1498-1506. doi:10.1111/j.1398-9995.2009.02093.x

8. Sattar HA, Mobayed H, al-Mohammed AA, et al. The pattern of indoor and outdoor respiratory allergens in asthmatic adult patients in a humid and desert newly developed country. Eur Ann Allergy Clin Immunol. 2003;35(8):300-305.

9. Bener A, Ehlayel M, Sabbah A. The pattern and genetics of pediatric extrinsic asthma risk factors in polluted environment. Eur Ann Allergy Clin Immunol. 2007;39(2):58-63.

10. Kerkadi A, Althani A, Boainain A, Singh R. Sensitization to common food and inhalant allergens in children attending allergy and asthma pediatric clinic in Doha, Qatar. $J$ Food Agric Environ. 2009;7:213-217.

11. Dawod ST, Hussain AA. Childhood asthma in Qatar. Ann Allergy Asthma Immunol. 1995;75(4):360-364.

12. Janahi IA, Bener A, Bush A. Prevalence of asthma among Qatari schoolchildren: International Study of asthma and allergies in childhood, Qatar. Pediatr Pulmonol. 2006;41(1):80-86. doi:10.1002/ ppul.20331

13. Sharif H, Salih E, Awad H, Albarazi R. Common aeroallergens among children with allergies in the United Arab Emirates. Indian $J$ Allergy Asthma Immunol. 2018;32:4-9. doi:10.4103/ijaai. ijaai_32_17

14. Liu T, Valdez R, Yoon PW, Crocker D, Moonesinghe R, Khoury MJ. The association between family history of asthma and the prevalence of asthma among US adults: National Health and Nutrition Examination Survey, 1999-2004. Genet Med. 2009;11(5):323-328. doi:10.1097/GIM.0b013e31819d3015
15. Al-Rabia MW. Prevalence and severity of sensitization reaction to food and inhalant allergens among allergic patients from Jeddah city: Saudi Arabia. Int J Community Med Public Health. 2016;3 (10):2908-2915. doi:10.18203/2394-6040.ijcmph20163382

16. Almogren A. Airway allergy and skin reactivity to aeroallergens in Riyadh. Saudi Med J. 2009;30(3):392-396.

17. AlKhater SA. Sensitization to common aeroallergens in asthmatic children in the Eastern region of Saudi Arabia. Saudi J Med Med Sci. 2017;5(2):136-141. doi:10.4103/1658-631X.204876

18. Aburuz S, Bulatova N, Tawalbeh M. Skin prick test reactivity to aeroallergens in Jordanian allergic rhinitis patients. East Mediterr Health J. 2011;17(7):604-610. doi:10.26719/2011.17.7.604

19. Moghtaderi M, Hejrati Z, Kolahi N, Heidari B. Sensitization to aeroallergens in patients with allergic rhinitis, asthma, and atopic dermatitis in Shiraz, Southwestern Iran. Indian J Allergy Asthma Immunol. 2015;29(2):79-83. doi:10.4103/0972-6691.178272

20. Moghtaderi M, Hosseini Teshnizi S, Farjadian S. Sensitization to common allergens among patients with allergies in major Iranian cities: a systematic review and meta-analysis. Epidemiol Health. 2017;39. doi:10.4178/epih.e2017007

\section{Publish your work in this journal}

The Journal of Asthma and Allergy is an international, peer-reviewed open-access journal publishing original research, reports, editorials and commentaries on the following topics: Asthma; Pulmonary physiology; Asthma related clinical health; Clinical immunology and the immunological basis of disease; Pharmacological interventions and new therapies. The manuscript management system is completely online and includes a very quick and fair peer-review system, which is all easy to use. Visit http://www.dovepress.com/testimonials.php to read real quotes from published authors. 